\title{
Embryos under the X-ray
}

X-ray imaging using synchrotron radiation reveals the cellular choreography of a developing frog embryo in spectacular detail.

The African claw-toed frog may not be a beauty, but its plentiful eggs have helped generations of developmental biologists study the mechanisms that pattern young animal tissues. In particular, it has been used to understand gastrulation, the coordinated shuffling of cells into three primary layers that ultimately organize the tissues and organs of the adult. But the Achilles' heel of studies using this organism-the frog's opaque embryo-makes light microscopy off-limits for live animal imaging.

To visualize cell dynamics deep inside living embryos at high resolution, Jubin Kashef and Ralf Hofmann at the Karlsruhe Institute of Technology took X-ray movies.

$\mathrm{X}$-ray imaging is a familiar way to probe internal structures, with applications from tooth decay to broken bones, and modern methods enable doctors to image continuously during surgery or dye tracing procedures. X-rays form a silhouetted image of tissues that absorb or scatter the most radiation. Absorptive imaging, however, typically requires contrast agents and high doses of radiation to scan soft tissues. It cannot resolve gastrulation in embryos because of their low differential absorption.

With an international team of physicists and biologists, Kashef and Hofmann used X-ray diffraction to overcome these limitations. "Normally in medicine you get maybe a millimeter in spatial resolution," says Hofmann. "We get about 1,000 times better resolution." X-ray diffraction relies on the use of a synchrotron source for highquality, coherent light. Coherent light waves travel in the same phase, so the differential slowing caused by transiting various tissues produces distinctive diffraction patterns of the object. The object is continually rotated to generate a series of two-dimensional (2D) diffraction patterns at different angles. "These interference effects are used for con-

trast formation that you can mathematically invert to know what happened after [the x-rays left] the object. Once you know that, and you know it for various angles, you can $3 \mathrm{D}$ - reconstruct the object," says Hofmann. Reconstructing 3D
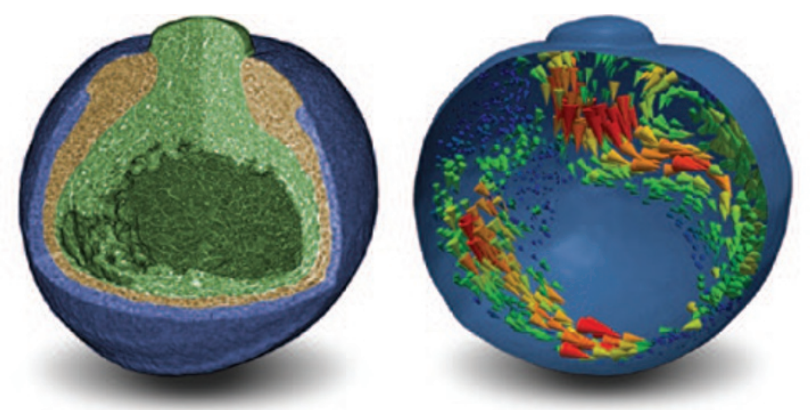

A frog embryo imaged by X-ray tomography (left), and a flow reconstruction of its cellular movements over time (right).

images from 2D sections is known as tomography.

Tissues are damaged by high-energy $\mathrm{X}$-ray radiation, making a full-length feature film of gastrulation a challenging prospect. Hofmann explains that the high intensity of the light allows for short exposures that prevent motion blurring. The researchers performed scans every 10 minutes at a single detection distance to minimize the splitting of water, which forms harmful radicals. Embryos did not heat up from the $\mathrm{X}$-ray exposure and appeared normal during the imaging window, beyond which tissues began eroding. It is also possible to optimize the method for longer imaging at lower resolution or for very high-resolution imaging of brief processes of interest.

The researchers discovered new embryonic structures from the dense tomographic data, including a ridge of tissue that may direct aspects of gastrulation. The ridge would not likely be found in dissected tissue, in which important structures that maintain tension are severed. Another strength of the work is in the global view the researchers took of morphogenetic processes: to better understand what propels cells, the team reconstructed velocity data using differential flow fields that describe changes in movement. "The spatial gradient of these velocity fields tells you about collectiveness of the motion ... what are the centers that drive motion," says Hofmann.

The method also allowed the researchers to image cavities and fluid redistribution in the embryo with high precision, and the results fended off a recent challenge to the idea that the primitive gut expands by imbibing water from the environment rather than from a fluid-filled cavity that forms earlier in the embryo.

Phase-contrast X-ray tomography is not for everyone or everything. "Anything with bony structures is not so good: you get artifacts introduced by absorptive effects," says Hofmann. Samples need to be around 1 centimeter thick or less and need to withstand the $\mathrm{X}$-ray radiation, the biological effects of which Hofmann says have not been studied exhaustively.

More importantly, the need for a synchrotron beam limits research. Presently, only eight facilities in the world can accommodate these types of experiments. Enormous maintenance costs, in addition to the cost of initial construction, will realistically keep their numbers low. Hofmann believes that table-top plasma laser devices, although they currently do not produce sufficiently coherent and intense X-rays, may eventually offer a good alternative. In the meantime, synchrotrons will hopefully continue to allot beam time to illuminate the intricate workings of small living organisms and embryos that have been difficult to image.

Tal Nawy

\section{RESEARCH PAPERS}

Moosmann, J. et al. X-ray phase-contrast in vivo microtomography probes new aspects of Xenopus gastrulation. Nature 497, 374-377 (2013). 